\title{
Interference Analysis Between Crack and General Inclusion in an Infinite Plate by Body Force Method
}

\author{
Takuichiro Ino ${ }^{1, a}$, Shohei Ueno ${ }^{1, b}$ and Akihide Saimoto ${ }^{2, c}$ \\ ${ }^{1}$ Graduate Student, Mechanical Engineering Course, Graduate School of Engineering, \\ Nagasaki University, 1-14 Bunkyo-machi, Nagasaki 852-8521, Japan \\ ${ }^{2}$ Corresponding Author: Graduate School of Engineering, Nagasaki University

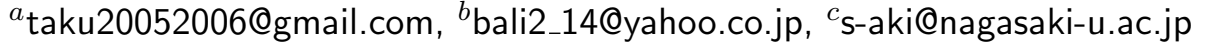

Keywords: Inclusion, Graded Material, Force doublet, Principle of superposition

\begin{abstract}
A continously embedded force doublet over the particular region can be regarded as the distributing eigen strain. This fact implies that many sorts of inelastic strain can be replaced by the force doublet. In the present paper, the force doublet is used to alter the local constitutive relationship. As a result, a method for analyzing the general inclusion problem in which the material properties of the inclusion are not only different from those of the matrix material but also can be even a function of spacial coordinate variables is proposed. The theoretical background of the present analysis is explained followed by some representative numerical results.
\end{abstract}

\section{Introduction}

There are at least two advantages, if the Body Force Method (BFM) is employed rather than the conventional Finite Element Method (FEM), for the analysis of graded materials. The 1st advantage is that, there is no need to prepare the mesh division to the domain where the material composition is identical to the matrix while the complete discretization of the total body is indispensable in FEM. The 2nd advantage is that the location of crack is admitted only along the boundary of each element in FEM while there is no such restriction in BFM.

So far, the inclusion problems analyzed by BFM were such a primitive one that the material composition or property of the inclusion region is uniform though the value is different from that of surrounding matrix material. M. Yatsuda, Y. Murakami and M. Isida[1] analyzed the stress distribution in the vicinity of an elliptic inclusion embedded in the infinite plate under remote tension. The Young's modulus and the Poisson's ratio in the inclusion and the matrix regions were uniform at $\left(E_{I}, \nu_{I}\right)$ and $\left(E_{M}, \nu_{M}\right)$ respectively. The most characteristic point of their approach was the use of the elastic fields due to a point force acting in a uniform infinite medium that corresponds the matrix and the inclusion regions independently. To the contrast, in the present analysis, the force doublet is employed in order to express the presence of general inclusion whose composition can be even a function of the spacial coordinate variables as in graded materials. Based on the present strategy, the interference problem between a general inclusion and a line crack and so on were computed and the results were shown graphically.

\section{Theoretical background}

The strategy for the treatment of general inclusion problem is illustrated in Fig.1. In the present analysis, the stress component at an arbitrary point $P$ in the matrix region $\left(\sigma_{i j}^{M}(P)\right)$ and that of in the inclusion region $\left(\sigma_{i j}^{I}(P)\right)$ is expressed by a superposition of the influence due 
to remote stresses $\sigma_{i j}^{\infty}(P)$ and the influence of force doublet of magnitude $T_{i j}(Q)$ embedded at a point $Q[2,3]$.

$$
\begin{aligned}
\sigma_{i j}^{M}(P) & =\sigma_{i j}^{\infty}(P)+\int_{\Omega} \sigma_{i j}^{k \ell}(P, Q) T_{k \ell}(Q) d \Omega(Q) \\
\sigma_{i j}^{I}(P) & =\sigma_{i j}^{\infty}(P)+\int_{\Omega} \sigma_{i j}^{k \ell}(P, Q) T_{k \ell}(Q) d \Omega(Q)-T_{i j}(P)
\end{aligned}
$$

As in a same manner, the strain components corresponding to the matrix $\left(\varepsilon_{i j}^{M}(P)\right)$ and the inclusion $\left(\varepsilon_{i j}^{I}(P)\right)$ region can be expressed as

$$
\varepsilon_{i j}^{M}(P)=\varepsilon_{i j}^{I}(P)=\varepsilon_{i j}^{\infty}(P)+\int_{\Omega} \varepsilon_{i j}^{k \ell}(P, Q) T_{k \ell}(Q) d \Omega(Q)
$$

In Eq.1 3, $i, j, k$ and $\ell$ are indices that express either of $x$ and $y . T_{x x}(Q), T_{y y}(Q)$ and $T_{x y}(Q)$ are unknown density of force doublet at point $Q$ to be embedded in the inclusion region $\Omega$ $(Q \in \Omega) . \quad \sigma_{i j}^{x x}(P, Q), \sigma_{i j}^{y y}(P, Q)$ and $\sigma_{i j}^{x y}(P, Q)$ are stress components at the reference point $P$ due to a unit magnitude of force doublet acting at the source point $Q$ into $x x$, yy and $x y$ directions, respectively. In a same manner, $\varepsilon_{i j}^{x x}(P, Q), \varepsilon_{i j}^{y y}(P, Q)$ and $\varepsilon_{i j}^{x y}(P, Q)$ are strain components at the reference point $P$ due to a unit magnitude of force doublet acting at the source point $Q$ into $x x, y y$ and $x y$ directions, respectively.

The constitutive equation between components of stress and strain follows

$$
\begin{gathered}
\sigma_{i j}^{M}(P)=D_{i j k \ell}^{M}(P) \varepsilon_{k \ell}^{M}(P), \varepsilon_{i j}^{M}(P)=C_{i j k \ell}^{M}(P) \sigma_{k \ell}^{M}(P) \\
\sigma_{i j}^{I}(P)=D_{i j k \ell}^{I}(P) \varepsilon_{k \ell}^{I}(P), \varepsilon_{i j}^{I}(P)=C_{i j k \ell}^{I}(P) \sigma_{k \ell}^{I}(P)
\end{gathered}
$$

where $D_{i j k \ell}^{M}$ and $C_{i j k \ell}^{M}$ are the stiffness and compliance tensor for matrix material and $D_{i j k \ell}^{I}$ and $C_{i j k \ell}^{I}$ are those for inclusion material. As seen in Eq.3, $\varepsilon_{i j}^{M}(P)$ and $\varepsilon_{i j}^{I}(P)$ has the identical expression. Therefore, the following relation is derived.

$$
C_{i j k \ell}^{I}(P) \sigma_{k \ell}^{I}(P)=C_{i j k \ell}^{M}(P) \sigma_{k \ell}^{M}(P)
$$

Eq. 6 defines the condition through which the unknown magnitude of body force doublet $T_{i j}(P)$ at point $P$ is determined as

$$
T_{i j}(P)=\left[E_{i j \alpha \beta}-D_{i j k \ell}^{I}(P) C_{k \ell \alpha \beta}^{M}(P)\right] \times\left[\sigma_{\alpha \beta}^{\infty}(P)+\int_{\Omega} \sigma_{\alpha \beta}^{s t}(P, Q) T_{s t}(Q) d \Omega(Q)\right]
$$

where $E_{i j \alpha \beta}$ is a unit matrix of order $3 \times 3$.
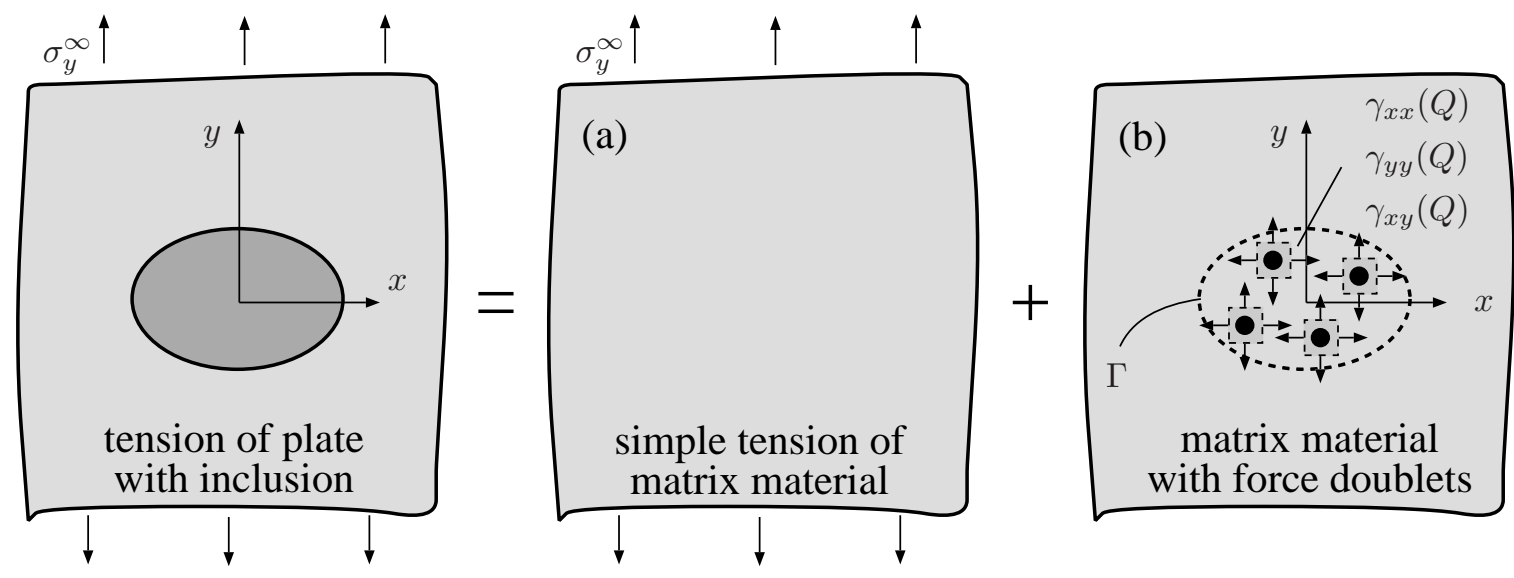

Figure 1: Analysis of inclusion problem by force doublets 


\section{Numerical example}

In order to verify the applicability of the present method, stress distribution in an infinite plate with a circular hole subjected to a uniform tensile stress at infinity was analyzed. A circular hole can be considered a kind of inclusion whose modulus of elasticity is zero. Fig.2 shows the analyzed $\sigma_{y y}$ distribution along the $x$ axis. In this analysis, the circular area corresponding to a circular hole was divided with regularly distributed number of triangles NT. As seen, the numerical solution exhibited a good agreement with theoretical value. Figure 3,4 and 5 shows the $\sigma_{y y}$ distribution along the $x$ axis for the cases of single circular inclusion (Fig.3), interference between a circular inclusion and a circular hole (Fig.4) and interference between a circular inclusion and a crack (Fig.5). The used number of triangles for the inclusion part was fixed at $\mathrm{NT}=1024$.

\section{Conclusion}

The body force doublet approach for solving the general inclusion problem was proposed. As the present method does not require any of special fundamental solution, an arbitrary inclusion problem can be solved in a same manner. For simplicity, the magnitude of force doublet for each triangular area was assumed at constant in the present study, however, higher order element could be easily introduced and expected to bring a further accurate solution.

\section{References}

[1] Yatsuda, A., Murakami, Y. and Isida, M., Stress field due to an interference of two elliptic inclusions, Journal of the Japan Society of Mechanical Engineers, Ser. A, 51-464, pp.1057-1065 (1985), in Japanese.

[2] Chen, D. H. and Nisitani, H., Extension of body force method to elastic-plastic problems, Journal of the Japan Society of Mechanical Engineers, Ser. A, 51-462, pp.571-578 (1985), in Japanese.

[3] Chen, D. H. and Nisitani, H., Stress field in a composite based on body force method, Journal of the Japan Society of Mechanical Engineers, Ser. A, 57-540, pp.1815-1821 (1991), in Japanese.
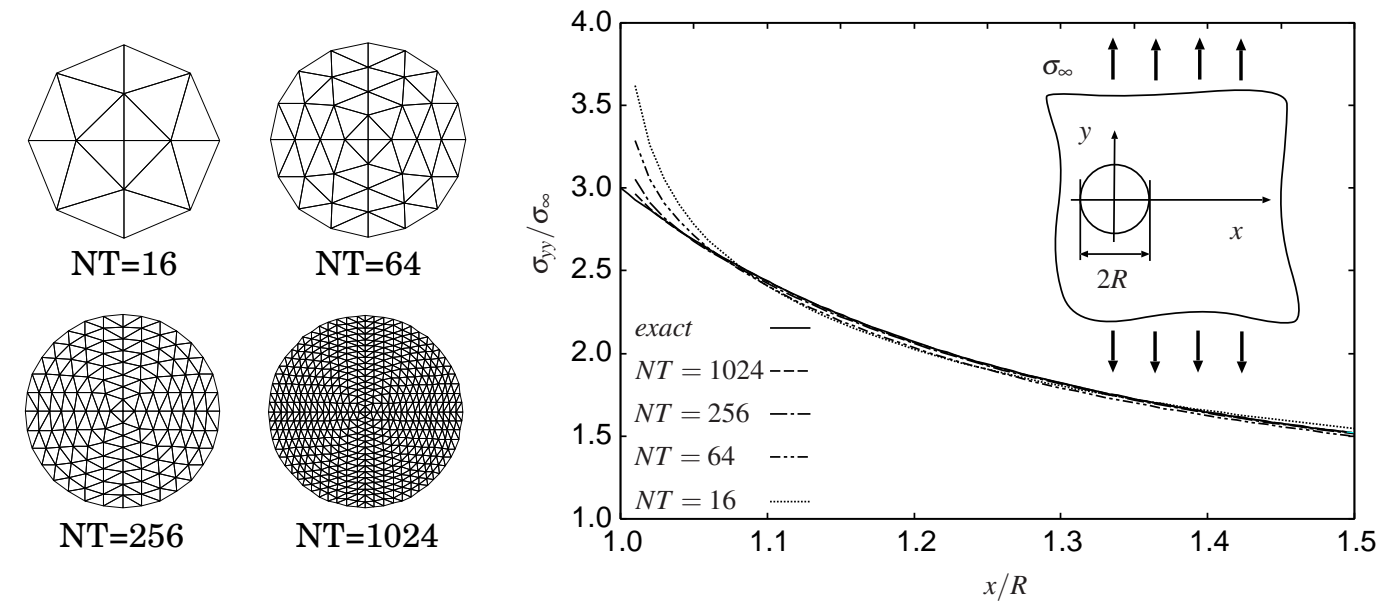

Figure 2: Triangular division of the circular area corresponding to a circular hole and the calculated $\sigma_{y y}$ distribution along the $x$ axis (NT: number of triangles) 

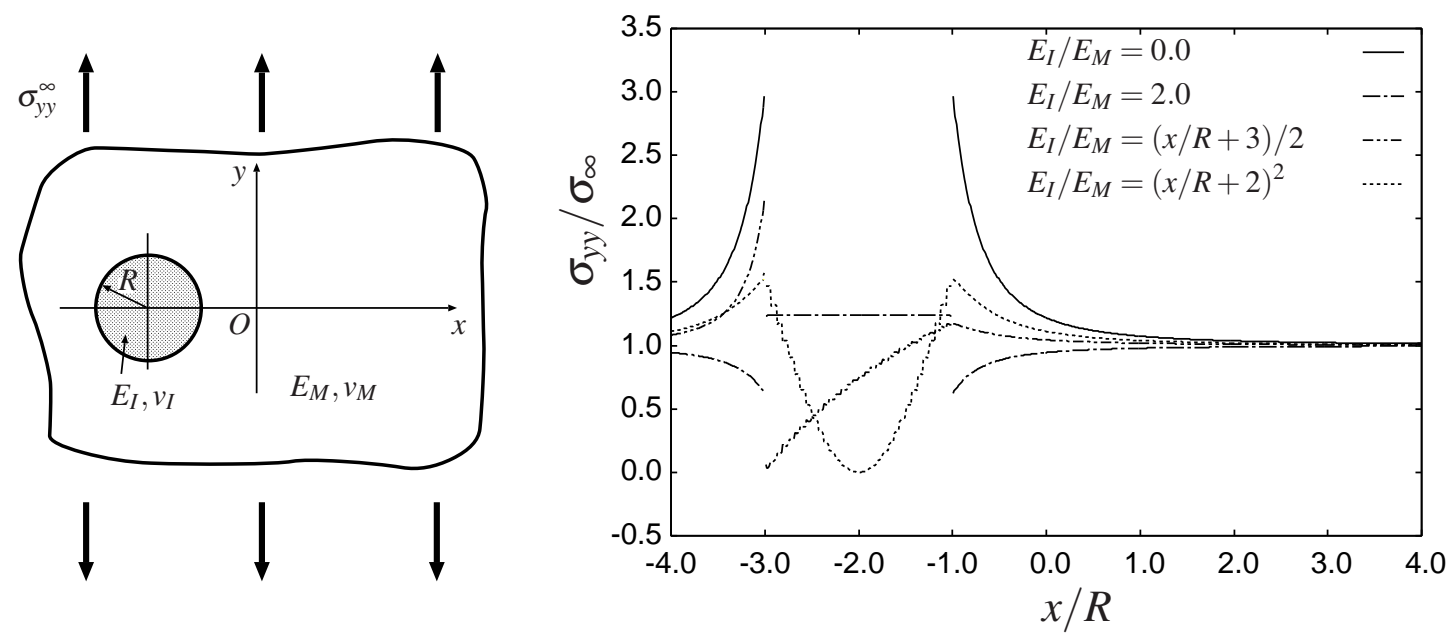

Figure 3: $\sigma_{y y}$ distribution along the $x$ axis for the problem of tension of an infinite plate with single circular inclusion of various characteristics $\left(\nu_{I}=\nu_{M}=0.0\right)$
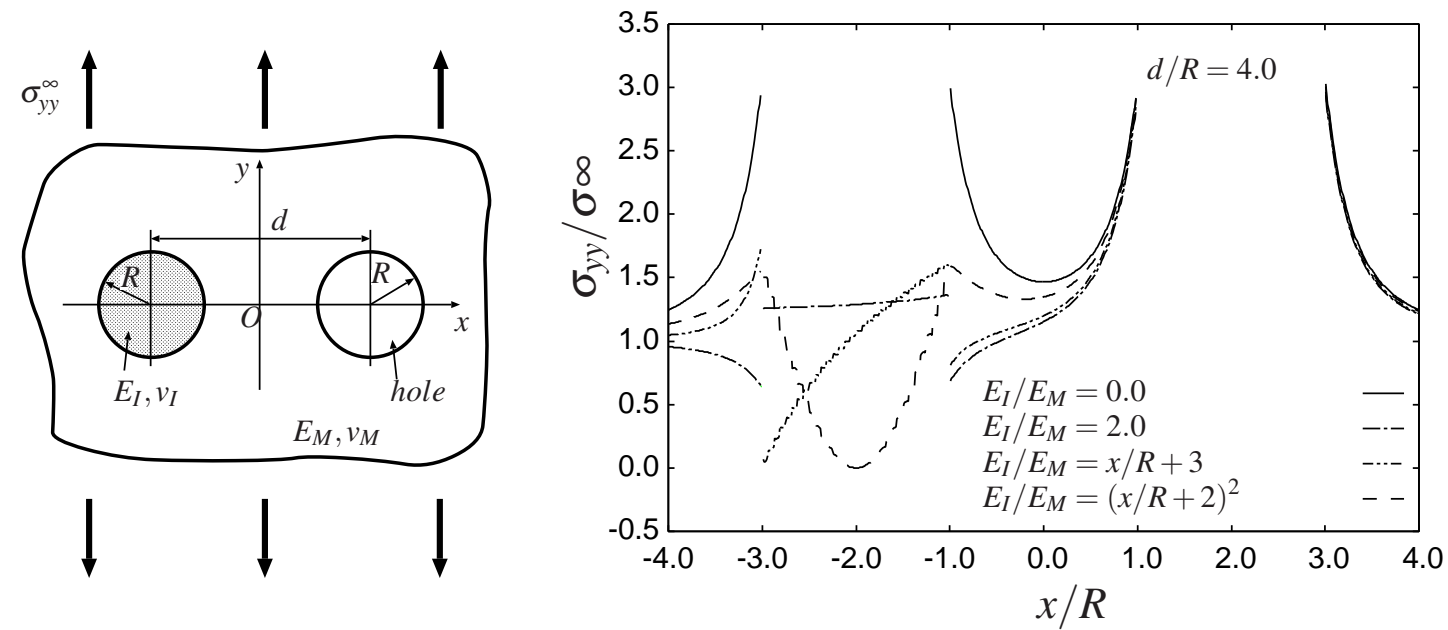

Figure 4: $\sigma_{y y}$ distribution along the $x$ axis for the problem of tension of an infinite plate with circular inclusion of various characteristics $\left(\nu_{I}=\nu_{M}=0.0\right)$ and a circular hole
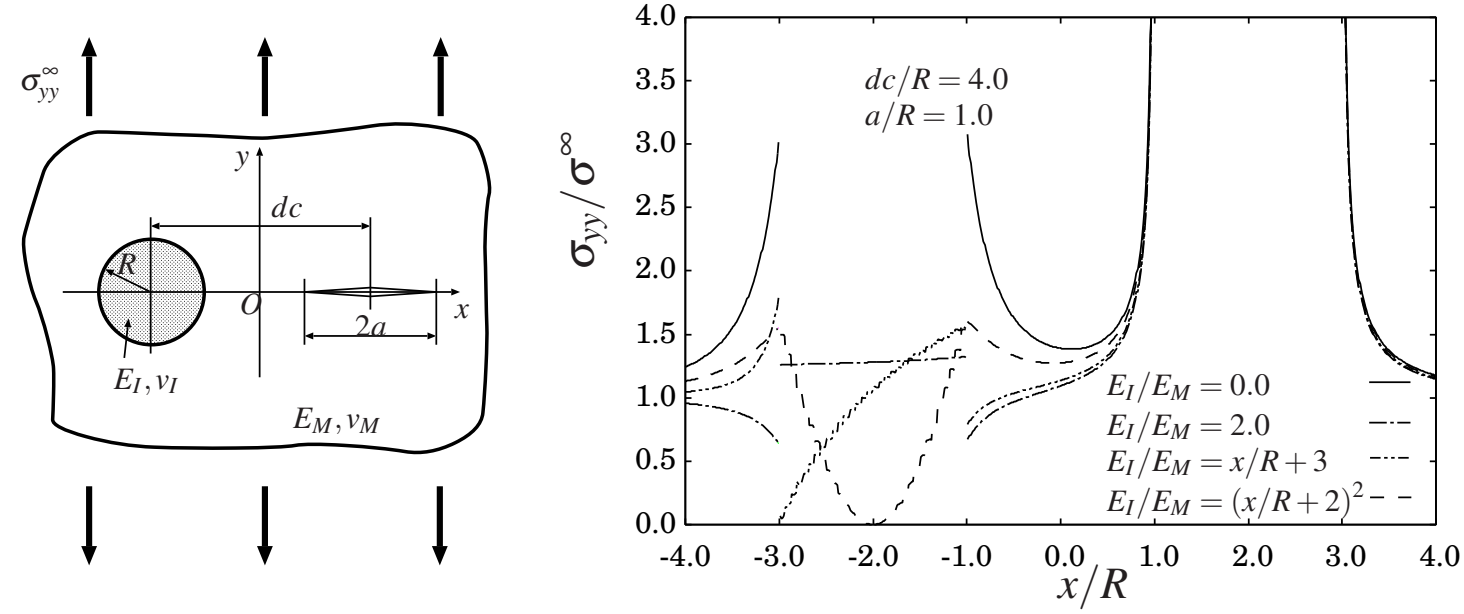

Figure 5: $\sigma_{y y}$ distribution along the $x$ axis for the problem of tension of an infinite plate with circular inclusion of various characteristics $\left(\nu_{I}=\nu_{M}=0.0\right)$ and a crack 